\title{
Clinical Study \\ The Influence of Pharmacological Preconditioning with Sevoflurane on Incidence of Early Allograft Dysfunction in Liver Transplant Recipients
}

\author{
Andrei F. Minou, ${ }^{1}$ Alexander M. Dzyadzko, ${ }^{1}$ Aliaksei E. Shcherba, ${ }^{2}$ and Oleg O. Rummo ${ }^{2}$ \\ ${ }^{1}$ Department of Anesthesiology and Critical Care, Republican Center of Organ and Tissue Transplantation, \\ Semashko Street 8, 220116 Minsk, Belarus \\ ${ }^{2}$ Department of Transplantology, Republican Center of Organ and Tissue Transplantation, Semashko Street 8, 220116 Minsk, Belarus
}

Correspondence should be addressed to Andrei F. Minou, andrei.minou@gmail.com

Received 15 June 2012; Accepted 2 July 2012

Academic Editor: Gebhard Wagener

Copyright (C) 2012 Andrei F. Minou et al. This is an open access article distributed under the Creative Commons Attribution License, which permits unrestricted use, distribution, and reproduction in any medium, provided the original work is properly cited.

Background. Pharmacological preconditioning is one of the tools used to diminish preservation injury. We investigated the influence of sevoflurane preconditioning of liver grafts on postoperative graft function. Methods. Consecutive 60 deceased brain donors were randomized into sevoflurane group or control group. In sevoflurane group donors were treated with endexpiratory 2,0 volume $\%$ of sevoflurane during procurement. Primary endpoint was postoperative liver injury. Secondary endpoint was incidence of early allograft dysfunction (EAD). Results. The groups were not different in median DRI, donor age, graft steatosis, and MELD score. Peak AST and ALT levels were lower in sevoflurane group than in control group: 792 and $1861(P=0,038)$ for AST and 606 and 1191 for ALT $(P=0,117)$. Incidence of EAD was 16,7\% in sevoflurane group and 50\% in control group (Fisher test, $P=0,013)$. In subgroups without steatosis preconditioning with sevoflurane did not have influence on incidence of EAD. In subgroups with mild and moderate steatosis incidence of EAD was lower in recipients of liver grafts treated with sevoflurane. Conclusions. Preconditioning with sevoflurane during organ procurement improves graft function by lowering incidence of early allograft dysfunction, particularly in recipients of steatotic liver grafts.

\section{Introduction}

In order to accommodate the growing list of patients awaiting liver transplantation, the transplant community has increased efforts to expand the donor pool by utilization of extended criteria donor organs $[1,2]$, which include organs distinguished by hepatic steatosis, old donor age, prolonged cold ischemia, or donation after cardiac death. These grafts are susceptible to preservation injury and as a consequence are prone to a higher incidence of early allograft dysfunction (EAD) [3]. Moreover, severe ischemia/reperfusion injury (IRI) significantly impacts transplantation outcome because it is a major risk factor for both early graft failure and late chronic allograft dysfunction.

Pharmacological preconditioning is one of the tools used to diminish preservation injury. Although volatile anesthetics and propofol have been studied to attenuate injury in liver resections with inflow occlusion $[4,5]$, pharmacological preconditioning with sevoflurane of human liver grafts from deceased brain donors has not yet been described. The purpose of this study was to evaluate the influence of sevoflurane preconditioning of liver grafts from deceased brain donors on postoperative graft function in patients undergoing liver transplantation.

\section{Materials and Methods}

Deceased brain liver donors were assessed for study eligibility to enroll overall number of 60 donors (30 in each group). Exclusion criteria were age less than 18 years and utilization of the procured graft for retransplantation or reduced size liver transplantation. Between November 2010 and December 2011 consecutive 67 deceased brain donors were assessed of whom 7 were excluded as they fell under exclusion criteria. 
Enrolled donors were randomized at the beginning of the procurement procedure into a sevoflurane group (inhaled anesthesia with sevoflurane) or a control group (without any volatile anesthetic). All other medication and surgical management was the same. The randomization sequence without any stratification was generated by computer and sealed with consecutively numbered envelopes providing concealment of random allocation. The study was approved by local ethics committee. Written informed consent was obtained from all recipients before liver transplantation.

At the beginning of procurement procedure all donors received bolus of $3 \mu \mathrm{g} / \mathrm{kg}$ fentanyl to blunt spinal reflexes and bolus of $12 \mathrm{mg}$ pipecuronium bromide for muscle relaxation. Electrocardiogram, arterial oxygen saturation, central venous pressure, and radial arterial blood pressure were monitored routinely. To maintain mean arterial pressure higher than $60 \mathrm{~mm} \mathrm{Hg}$ infusion of norepinephrine 0,05$0,15 \mu \mathrm{g} / \mathrm{kg} / \mathrm{min}$ was administered as indicated. In sevoflurane group pharmacological preconditioning with end-expiratory sevoflurane of 2,0 volume $\%$ in mixture of oxygen and air $\left(\mathrm{FiO}_{2}=0,4\right)$ was performed for the entire procedure of organ procurement. In control group donors inhaled only mixture oxygen and air $\left(\mathrm{FiO}_{2}=0,4\right)$ without any volatile anesthetic.

All organ procurement procedures were performed in standardized manner by 3 experienced transplant surgeons, who were blinded to randomization. After laparotomy in all donors liver specimen was taken for evaluation for the presence of steatosis. Liver biopsies were evaluated by a single pathologist for the presence of macrovesicular steatosis. Using haematoxylin and eosin-stained sections, the degree of macrovesicular steatosis was graded as absent $(0 \%)$, mild (1-30\%), or moderate (31-60\%) based on the percentage of hepatocytes with fat droplets.

All donor organ allografts were implanted by caval replacement technique with bicaval anastomoses. The anesthetic technique was the same in all recipients. Maintenance of anesthesia was obtained with sevoflurane (the minimal alveolar concentration was between 0,7 and 0,9). Fentanyl was given as continuous infusion. During a hepatic phase and after reperfusion norepinephrine $0,1-0,3 \mu \mathrm{g} / \mathrm{kg} / \mathrm{min}$ was used in all recipients to maintain mean arterial pressure at more than $60 \mathrm{~mm} \mathrm{Hg}$. Patients were ventilated with a fraction of inspired oxygen of 0,4 to 0,6 , tidal volumes of 7 to $10 \mathrm{~mL} / \mathrm{kg}$, and a positive end-expiratory pressure of 0 to $5 \mathrm{~cm} \mathrm{H}_{2} \mathrm{O}$. Transfusion triggers were similar, with a target hematocrit of 25 to 30 . Fresh frozen plasma, cryoprecipitate, and platelet concentrate were administered at the discretion of the attending anesthesiologist and according to results of thromboelastometry. All patients received intraoperative $500 \mathrm{mg}$ methylprednisolone as induction of immunosuppression. Transplant surgeons and anesthesiologists were blinded to randomization.

Primary endpoint was postoperative liver injury assessed by peak serum values of alanine aminotransferase (ALT) and aspartate aminotransferase (AST). Secondary endpoint was incidence of early allograft dysfunction (EAD) defined as the presence of one or more of the following criteria: bilirubin greater or equal $10 \mathrm{mg} / \mathrm{dL}$ on day 7 , international normalized ratio (INR) greater than or equal to 1,6 on day 7 , and ALT or AST greater than $2000 \mathrm{IU} / \mathrm{L}$ within the first 7 days after liver transplantation [6]. We performed limited number of subgroup analyses to assess the influence of degree of macrovesicular steatosis on protective effect of pharmacological preconditioning with sevoflurane.

Group sample size was calculated based on two assumptions. In our previous experience the mean postoperative peak levels of AST and ALT were $2532 \pm 1013 \mathrm{IU} / \mathrm{L}$ and $1561 \pm 625 \mathrm{IU} / \mathrm{L}$, respectively. We expected that pharmacological preconditioning with sevoflurane could produce $30 \%$ reduction in peak levels of liver transaminases, as it was observed by Beck-Schimmer et al. [4]. Thus, to achieve a 30\% reduction in peak levels of transaminases with an $\alpha$ error of 0,05 and a power $0,80,29$ patients were needed in both groups. The Shapiro-Wilk test was used to check for normal distribution. Nonparametric data were expressed as median (interquartile range) and parametric data as mean \pm SD. Group means were compared using Mann-Whitney $U$-test or Student's $t$-test as appropriate. Categorical variables were compared using two-tailed Fisher's exact test. Significance was defined as $P<0,05$. Calculations were made using SPSS 18.0 software (SPSS Inc., Chicago, IL, USA).

\section{Results}

Thirty liver grafts from deceased brain donors were included in each group. Donor demographics, as well as cold and warm ischemia time, percentage of steatosis, and donor risk injury (DRI) score (calculated as described by Feng et al. [7]) are presented in Table 1. None of these parameters were statistically different.

Table 2 shows the recipient characteristics: age, MELD score, baseline levels of ALT, and AST. There were no significant differences in recipient characteristics between sevoflurane and control groups. No one in both groups had severe comorbidities such as coronary artery disease, chronic obstructive pulmonary disease, or diabetes mellitus.

The degree of IRI of the liver was assessed by postoperative peak serum ALT and AST levels. The peak of the transaminases occurred between 24 and 48 hours after surgery. The peak levels of AST and ALT were lower in sevoflurane group compared to the control group, but the difference was statistically significant only for peak level of AST (Table 3). The incidence of early allograft dysfunction (EAD) was lower in sevoflurane group (16,7\%) compared with control group (50\%). No patient experienced primary graft nonfunction in both groups. Despite higher incidence of EAD in control group, there were no significant differences in length of ICU and hospital stay between two groups (Table 3).

We performed limited number of subgroup analyses to further investigate the influence of degree of macrovesicular steatosis on the protective effects of pharmacological preconditioning with sevoflurane. The two groups were almost identical with respect to numbers of liver grafts without steatosis, with mild and moderate macrovesicular steatosis (Table 4). 
TABle 1: Donor characteristics.

\begin{tabular}{lccc}
\hline & Sevoflurane group & Control group & Mann-Whitney, $P$ value \\
\hline Donor age, $y$ & $32(24-46)$ & $39(25-46)$ & 0,515 \\
Weight, kg & $75(63,5-80)$ & $75,5(70-85)$ & 0,596 \\
BMI, kg/m & $24,2(22,8-25,9)$ & $24,8(21,9-26,8)$ & 0,744 \\
Cold ischemia time, min & $455(365-565)$ & $465(400-585)$ & 0,329 \\
Warm ischemia time, min & $62,5(60-70)$ & $67,5(60-70)$ & 0,623 \\
Percentage of macrovesicular steatosis, $\%$ & $15(0-20)$ & $10(0-20)$ & 0,922 \\
DRI score & $1,19(1,03-1,25)$ & $1,23(1,11-1,38)$ & 0,186 \\
\hline
\end{tabular}

Data are as median (interquartile range). BMI: body mass index, DRI: donor risk injury.

TABLE 2: Recipient characteristics.

\begin{tabular}{lccc}
\hline & $\begin{array}{c}\text { Sevoflurane } \\
\text { group }\end{array}$ & $\begin{array}{c}\text { Control } \\
\text { group }\end{array}$ & $\begin{array}{c}\text { Mann-Whitney, } \\
P \text { value }\end{array}$ \\
\hline Recipient age, y & $44(28-55)$ & $49(34-55)$ & $0,865^{*}$ \\
MELD score & $20(15-27)$ & $17(14-26)$ & $0,340^{*}$ \\
Baseline AST, IU/L & $86(32-154)$ & $90(34-171)$ & $0,955^{*}$ \\
$\begin{array}{l}\text { Baseline ALT, IU/L } \\
\begin{array}{l}\text { Beta-blocker } \\
\text { therapy, y/n }\end{array}\end{array}$ & $17 / 13$ & $15 / 15$ & $0,985^{*}$ \\
\hline
\end{tabular}

Data are as median (interquartile range).

*Mann-Whitney test, ** Fisher's exact test, two tailed.

TABLe 3: The influence of pharmacological preconditioning with sevoflurane on graft function and length of ICU and hospital stay.

\begin{tabular}{lccc}
\hline & $\begin{array}{c}\text { Sevoflurane } \\
\text { group }\end{array}$ & Control group & $P$ value \\
\hline Peak AST, IU/L & $792(481-1436)$ & $1861(519-3590)$ & $0,038^{*}$ \\
Peak ALT, IU/L & $606(344-892)$ & $1191(392-2137)$ & $0,117^{*}$ \\
$\begin{array}{l}\text { Incidence of EAD, \% } \\
\text { Length of ICU stay, d }\end{array}$ & $6(5-7$ of 30$)$ & $50,0(15$ of 30$)$ & $0,013^{* *}$ \\
$\begin{array}{l}\text { Length of hospital } \\
\text { stay, d }\end{array}$ & $18(14-22)$ & $6(4-9)$ & $0,655^{*}$ \\
\hline
\end{tabular}

Data are as median (interquartile range). AST: aspartate aminotransferase, ALT: alanine aminotransferase, EAD: early allograft dysfunction, ICU: intensive care unit.

*Mann-Whitney test, ${ }^{* *}$ Fisher's exact test, two tailed.

TABLE 4: Number of grafts without and with mild and moderate macrovesicular steatosis.

\begin{tabular}{lccc}
\hline & \multicolumn{3}{c}{ Macrovesicular steatosis } \\
& $\begin{array}{c}\text { None } \\
(0 \%)\end{array}$ & $\begin{array}{c}\text { Mild } \\
(1-30 \%)\end{array}$ & $\begin{array}{c}\text { Moderate } \\
(31-60 \%)\end{array}$ \\
\hline Sevoflurane group, $n$ & 9 & 16 & 5 \\
Control group, $n$ & 9 & 15 & 6 \\
\hline
\end{tabular}

The pharmacological preconditioning with sevoflurane did not have influence on the IRI of grafts without macrovesicular steatosis. There were no significant differences in peak levels of aminotransferases between the two subgroups. In subgroups with mild macrovesicular steatosis the peak values of liver transaminases were lower in recipients of grafts treated with sevoflurane compared to recipients of grafts without any preconditioning, but the differences were not statistically significant. In subgroups with moderate macrovesicular steatosis the peak levels of AST and ALT were lower in recipients of grafts with sevoflurane preconditioning compared to recipients of grafts without preconditioning, but the difference was statistically significant only for peak level of AST (Table 5).

Effect of sevoflurane preconditioning on graft function depended on the degree of macrovesicular steatosis. In subgroups without macrovesicular steatosis pharmacological preconditioning with sevoflurane did not have influence on incidence of EAD. In subgroups with mild and moderate macrovesicular steatosis incidence of EAD was lower in recipients of liver grafts treated with sevoflurane, but the difference was statistically significant only in subgroup with mild steatosis (Table 6).

\section{Discussion}

We evaluated the influence of sevoflurane preconditioning of liver grafts from deceased brain donors on postoperative graft function in patients undergoing liver transplantation. This randomized controlled trial demonstrated the protective effects of pharmacological preconditioning with sevoflurane. Reperfusion injury assessed by peak serum levels of transaminases was attenuated in sevoflurane group. Preconditioning with sevoflurane improved graft function by lowering the incidence of early allograft dysfunction. The observed protective effects were seen only in recipients of grafts with macrovesicular steatosis and were absent in recipients of grafts without macrovesicular steatosis. Our data are consistent with results of the study by Beck-Schimmer et al. [4], in which protective effects of preconditioning with sevoflurane on IRI were more pronounced in patients with liver steatosis.

Postoperative liver graft function depends on many factors related to donor and recipients characteristics. The DRI developed by Feng et al. [7] is a continuous scoring system, which includes only donor and transplant parameters found to significantly influence outcomes after liver transplantation. The DRI was also validated for use within Eurotransplant region [8]. In our study the two groups were comparable with respect to DRI score and some parameters included in DRI: donor age and cold ischemia time. There were no differences between two groups in characteristics not included in DRI, but which could have influence on 
TABLE 5: The influence of degree of macrovesicular steatosis on IRI of grafts with and without pharmacological preconditioning.

\begin{tabular}{|c|c|c|c|c|}
\hline \multirow{2}{*}{ Macrovesicular steatosis } & \multicolumn{2}{|c|}{ AST } & \multicolumn{2}{|c|}{ ALT } \\
\hline & Sevoflurane & Control & Sevoflurane & Control \\
\hline None $(0 \%)$ & $669(276-1327)$ & $759(315-1021)$ & $421(285-974)$ & $413(222-603)$ \\
\hline Mild (1-30\%) & $825(515-1654)$ & $2571(524-3493)$ & $576(363-861)$ & $1666(481-2642)$ \\
\hline Moderate (31-60\%) & $979(658-2267)^{*}$ & $4002(2322-8601)$ & $757(428-1776)$ & $1711(940-3474)$ \\
\hline
\end{tabular}

Data are as median (interquartile range). AST: aspartate aminotransferase, ALT: alanine aminotransferase.

${ }^{*} P<0,05$ (Mann-Whitney $U$-test) versus control group.

TABle 6: The influence of degree of macrovesicular steatosis on protective effects of sevoflurane preconditioning.

\begin{tabular}{|c|c|c|c|}
\hline \multirow{2}{*}{$\begin{array}{l}\text { Macrovesicular } \\
\text { steatosis }\end{array}$} & \multicolumn{2}{|c|}{ Incidence of EAD, \% } & \multirow{2}{*}{$\begin{array}{l}\text { Fisher's exact test, } \\
\text { two-tailed } P \text { value }\end{array}$} \\
\hline & $\begin{array}{l}\text { Sevoflurane } \\
\text { group }\end{array}$ & $\begin{array}{c}\text { Control } \\
\text { group }\end{array}$ & \\
\hline None $(0 \%)$ & $11,1(1$ of 9$)$ & $11,1(1$ of 9$)$ & 1,000 \\
\hline Mild (1-30\%) & $18,8(3$ of 16$)$ & $60,0(9$ of 15$)$ & 0,029 \\
\hline $\begin{array}{l}\text { Moderate } \\
(31-60 \%)\end{array}$ & $20,0(1$ of 5$)$ & $83,3(5$ of 6$)$ & 0,080 \\
\hline
\end{tabular}

EAD: early allograft dysfunction.

graft function: warm ischemia time and percentage of macrovesicular steatosis. In 2010 Olthoff et al. [6] validated a current definition of EAD. In their study in the multivariable analysis only the age of the donor and MELD score were significantly associated with EAD. Neither donor age nor MELD score was statistically different in our study.

The development of strategies to counteract IRI of the liver is a major challenge in liver surgery and transplantation. Some of clinical strategies reported to ameliorate IRI in liver transplantation include donor treatment with steroids [9], caspase inhibition [10], ATG induction therapy [11], donor organ flush with calcineurin inhibitor [12], nitric oxide (NO) inhalation [13], and infusion of the donor liver with rPSGLIg [14]. Pharmacological preconditioning of liver grafts with sevoflurane is a new strategy, which can easily be applied during organ procurement procedure and lacks of serious adverse effects.

Despite the fact that preconditioning with sevoflurane is a new strategy for liver transplantation, it has been examined in other clinical situations, including liver surgery. There is growing evidence that pharmacological preconditioning with volatile anesthetics may provide a new and easily applicable therapeutic option to protect the liver from IRI. In the study by Imai et al. [15] isoflurane, sevoflurane, and halothane reduced IRI in isolated perfused rat liver when administered during the reperfusion phase; however, they did not reduce injury when administered only during ischemia. Imai et al. [15] suggested that volatile anesthetics might protect the fasted liver from early, neutrophilindependent IRI by acting during the reperfusion phase. The results of study by Ishida et al. [16] showed that the extent of the hepatic IRI seen under sevoflurane anesthesia in pigs did not differ significantly from that seen under isoflurane, as judged from measurements of a number liver damage markers over a $240 \mathrm{~min}$ reperfusion period. The study conducted by Bedirli et al. [17] suggested that clinically relevant concentrations of sevoflurane given before, during and after hepatic ischemia protected the liver in rats against IRI, whereas the effects of isoflurane on hepatic IRI were not notable. The study by Beck-Schimmer et al. [4] showed that sevoflurane preconditioning was protective against IRI during liver resection. Sevoflurane preconditioning was shown to prevent hepatic injury, defined by transaminase levels, and improve clinical outcome. In the volatile preconditioning group, the expression of inducible nitric oxide synthase upon reperfusion significantly increased compared with the baseline value, which points to a possible protective role of nitric oxide in pharmacological preconditioning. The observed protective effects were more pronounced in patients with liver steatosis. However, Song et al. [5] compared liver function after hepatectomy with inflow occlusion between sevoflurane and propofol anesthesia and found no significant differences in postoperative liver function, as measured by serial transaminase levels, or in clinical outcomes. Patients with biopsy-proven cirrhosis did not have worse postoperative liver function than those without cirrhosis likely because the period of ischemia was too short to have significant impact on liver function. Perhaps, longer ischemic stress could reveal differences between sevoflurane and propofol anesthesia. The study by Ko et al. [18] suggested better postoperative hepatic and renal function tests with desflurane than sevoflurane at equivalent dose of 1 minimum alveolar concentration in living donors undergoing right hepatectomy.

Our study had several limitations. Although the study suggested statistically significant differences between sevoflurane and control groups with respect to incidence of EAD, our study was underpowered as sample size, especially in subgroup analysis, and was relatively small. Therefore interpretation of subgroup analysis needs to be done carefully as reproducibility of these findings is low. Despite the fact that the statistical significance of our data is limited by the sample size, we believe that this is the first clinical trial to demonstrate a protective effect of sevoflurane preconditioning during organ procurement on graft function in liver transplantation.

\section{Conclusions}

Our data indicate that there is a beneficial effect of preconditioning with sevoflurane on liver graft function. Pharmacological preconditioning with sevoflurane during organ procurement improves graft function by lowering incidence 
of early allograft dysfunction, particularly in recipients of steatotic liver grafts.

\section{References}

[1] “HHS/HRSA/HSB/DOT OPTN / SRTR," Annual Data Report, pp. 54-58, 2010.

[2] N. R. Barshes, I. B. Horwitz, L. Franzini, J. M. Vierling, and J. A. Goss, "Waitlist mortality decreases with increased use of extended criteria donor liver grafts at adult liver transplant centers," American Journal of Transplantation, vol. 7, no. 5, pp. 1265-1270, 2007.

[3] B. Alkofer, B. Samstein, J. V. Guarrera et al., "Extended-donor criteria liver allografts," Seminars in Liver Disease, vol. 26, no. 3, pp. 221-233, 2006.

[4] B. Beck-Schimmer, S. Breitenstein, S. Urech et al., "A randomized controlled trial on pharmacological preconditioning in liver surgery using a volatile anesthetic," Annals of Surgery, vol. 248, no. 6, pp. 909-916, 2008.

[5] J. C. Song, Y. M. Sun, L. Q. Yang, M. Z. Zhang, Z. J. Lu, and W. F. Yu, "A comparison of liver function after hepatectomy with inflow occlusion between sevoflurane and propofol anesthesia," Anesthesia and Analgesia, vol. 111, no. 4, pp. 1036-1041, 2010.

[6] K. M. Olthoff, L. Kulik, B. Samstein et al., "Validation of a current definition of early allograft dysfunction in liver transplant recipients and analysis of risk factors," Liver Transplantation, vol. 16, no. 8, pp. 943-949, 2010.

[7] S. Feng, N. P. Goodrich, J. L. Bragg-Gresham et al., "Characteristics associated with liver graft failure: the concept of a donor risk index," American Journal of Transplantation, vol. 6, no. 4, pp. 783-790, 2006.

[8] J. J. Blok, A. E. Braat, R. Adam et al., "Validation of the donor risk index in orthotopic liver transplantation within the Eurotransplant region," Liver Transplantation, vol. 18, no. 1, pp. 113-120, 2012.

[9] K. Kotsch, F. Ulrich, A. Reutzel-Selke et al., "Methylprednisolone therapy in deceased donors reduces inflammation in the donor liver and improves outcome after liver transplantation a prospective randomized controlled trial," Annals of Surgery, vol. 248, no. 6, pp. 1042-1049, 2008.

[10] E. S. Baskin-Bey, K. Washburn, S. Feng et al., "Clinical trial of the pan-caspase inhibitor, IDN-6556, in human liver preservation injury," American Journal of Transplantation, vol. 7, no. 1, pp. 218-225, 2007.

[11] D. Bogetti, H. N. Sankary, T. M. Jarzembowski et al., "Thymoglobulin induction protects liver allografts from ischemia/reperfusion injury," Clinical Transplantation, vol. 19, no. 4, pp. 507-511, 2005.

[12] S. D. S. Peter, D. J. Post, M. I. Rodriguez-Davalos, D. D. Douglas, A. A. Moss, and D. C. Mulligan, "Tacrolimus as a liver flush solution to ameliorate the effects of ischemia/reperfusion injury following liver transplantation," Liver Transplantation, vol. 9, no. 2, pp. 144-149, 2003.

[13] J. D. Lang, X. Teng, P. Chumley et al., "Inhaled NO accelerates restoration of liver function in adults following orthotopic liver transplantation," Journal of Clinical Investigation, vol. 117, no. 9, pp. 2583-2591, 2007.

[14] R. W. Busuttil, G. S. Lipshutz, J. W. Kupiec-Weglinski et al., "RPSGL-Ig for improvement of early liver allograft function: a double-blind, placebo-controlled, single-center phase II study," American Journal of Transplantation, vol. 11, no. 4, pp. 786-797, 2011.
[15] M. Imai, S. Kon, and H. Inaba, "Effects of halothane, isoflurane and sevoflurane on ischemia-reperfusion injury in the perfused liver of fasted rats," Acta Anaesthesiologica Scandinavica, vol. 40, no. 10, pp. 1242-1248, 1996.

[16] H. Ishida, Y. Kadota, T. Sameshima, A. Nishiyama, T. Oda, and Y. Kanmura, "Comparison between sevoflurane and isoflurane anesthesia in pig hepatic ischemia-reperfusion injury," Journal of Anesthesia, vol. 16, no. 1, pp. 44-50, 2002.

[17] N. Bedirli, E. Ofluoglu, M. Kerem et al., "Hepatic energy metabolism and the differential protective effects of sevoflurane and isoflurane anesthesia in a rat hepatic ischemiareperfusion injury model," Anesthesia and Analgesia, vol. 106, no. 3, pp. 830-837, 2008.

[18] J. S. Ko, M. S. Gwak, S. J. Choi et al., "The effects of desflurane and sevoflurane on hepatic and renal functions after right hepatectomy in living donors," Transplant International, vol. 23, no. 7, pp. 736-744, 2010. 


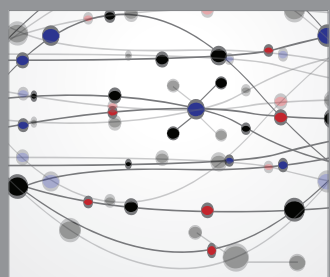

The Scientific World Journal
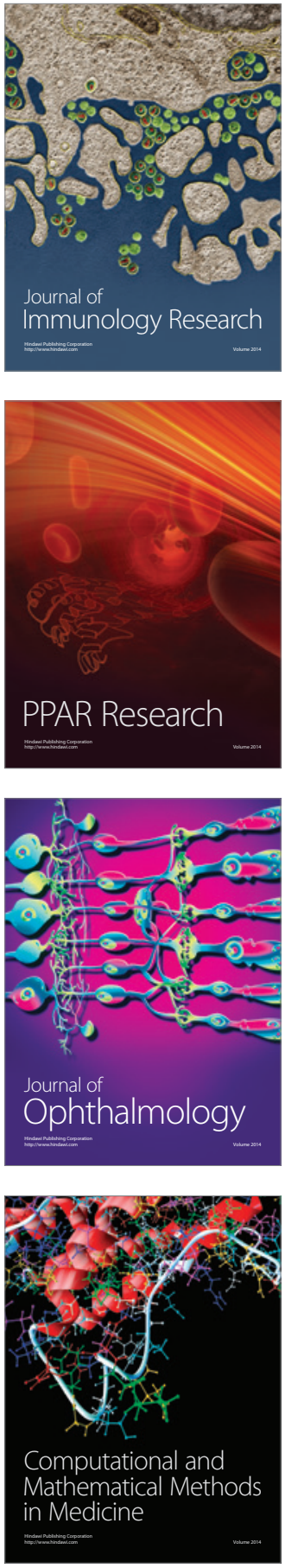

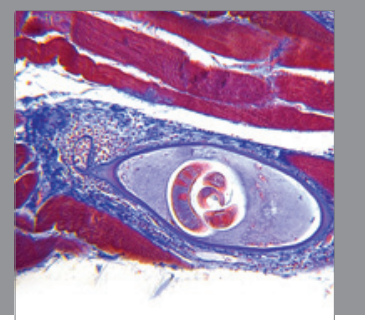

Gastroenterology

Research and Practice
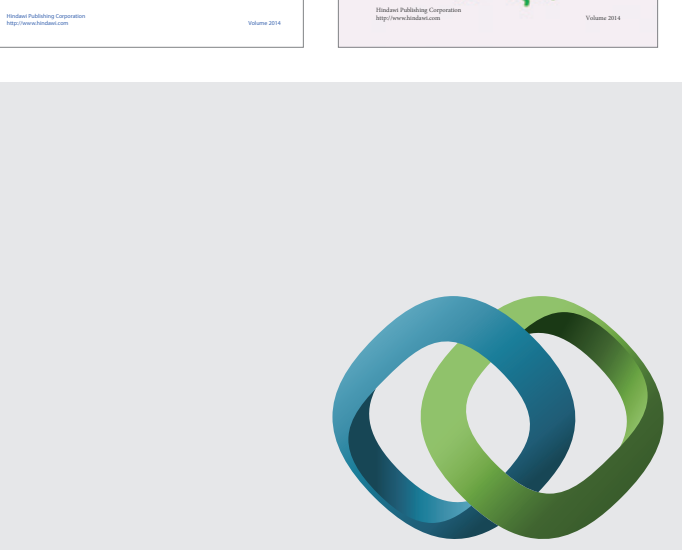

\section{Hindawi}

Submit your manuscripts at

http://www.hindawi.com
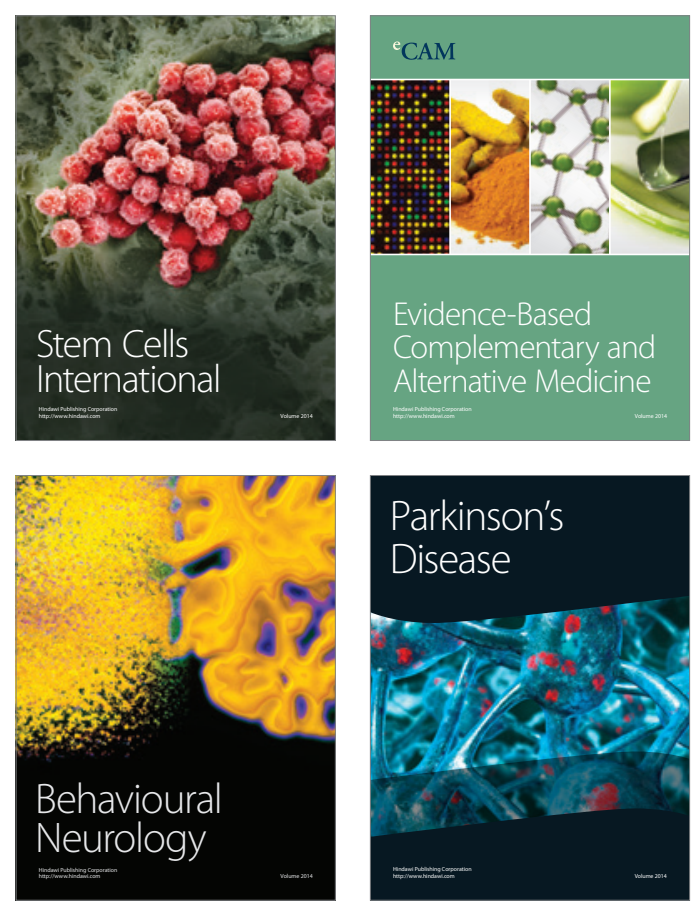

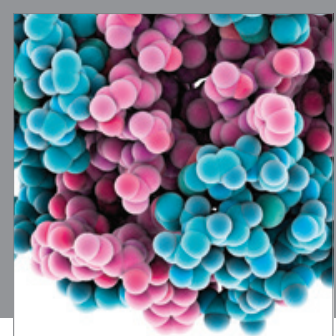

Journal of
Diabetes Research

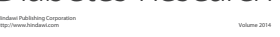

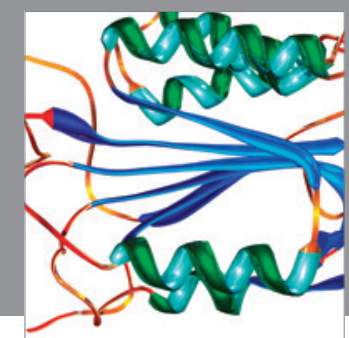

Disease Markers
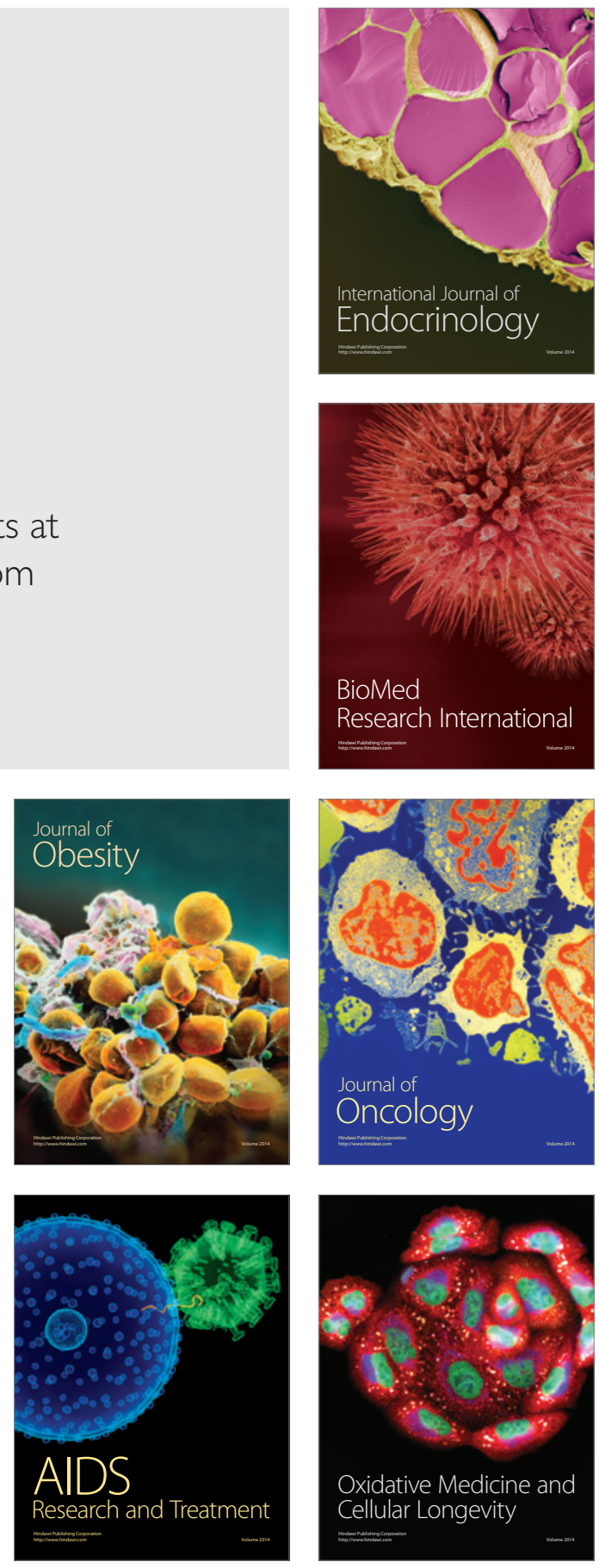